Sharif University of Technology
Scientia Iranica
SCIENTIA

\title{
A comparative study of various adaptive meshless methods for steady and unsteady flow calculations
}

\author{
S. Sattarzadeh and A. Jahangirian* \\ Department of Aerospace Engineering, Amirkabir University of Technology, 424 Hafez Avenue, Tehran, P.O. Box 15875-4413, Iran.
}

Received 6 September 2014; received in revised form 13 November 2014; accepted 16 February 2015

\section{KEYWORDS}

Meshless method; Adaptivity;

Navier-Stokes

equations;

Compressible flow;

Explicit method.

\begin{abstract}
Three different adaptive methods are presented for meshless calculation of steady and unsteady flows. Two approaches of point refinement/coarsening and point movement have their ground in the mesh-based methods that, in the present work, are extended for meshless calculations. However, the third approach is a new concept, socalled adaptive neighboring scheme, that concerns the optimum selection of the neighbors for each point in the meshless framework. This means that the selection of the neighboring region for each particular point is affected by the flow features in the domain. In this paper, an explicit meshless method based on the least square scheme is used. The results are presented for different steady and unsteady flows and the efficiency of the methods in terms of computational cost and accuracy is investigated. It is observed that using these adaptive approaches decrease the computational cost of the method by about $60 \%$ as compared with the un-adapted results while improving the accuracy of results at the same time.
\end{abstract}

(C) 2016 Sharif University of Technology. All rights reserved.

\section{Introduction}

In the recent years, researchers have tried to solve the flows with complex stationary and/or moving boundaries by using Euler and Navier-Stokes equations in different regimes. One of the main problems in the Computational Fluid Dynamics (CFD) for numerical flow simulation around complex geometries is quality of the mesh. Generating meshes with high quality, especially around complex geometries, are encouraging to develop meshless methods. In these methods, for each node, only the neighbors of that point are used. This property can decrease the dependency on the mesh (especially the connections), particularly around complex geometries.

Lohner has shown that generation of a point cloud distribution which can be used in meshless methods

*. Corresponding author. Tel.: +982164543223; E-mail addresses: sattarzadeh@aut.ac.ir (S. Sattarzadeh); ajahan@aut.ac.ir (A. Jahangirian) is obviously faster than the unstructured grids which are usually used in mesh-based methods [1]. Several meshless methods have been used [2-6] with different advantages and drawbacks. In these methods, approximation of the characteristics or derivatives is based on a group of nodes which can be nominated as neighbors. So, in most of these methods, the area or volume are not calculated at all, which may lead to lack of conservation. To overcome this problem and improve accuracy, higher-order methods are used [7]. Meshless methods need strong instrument in comparison with mesh-based methods [4]. To decrease the computational cost, convergence accelerating techniques are applied [8]. Explicit method is usually used for time discretization $[5,6]$. In the work by Katz and Jameson, it is shown that Taylor series least-square method has better accuracy than radial basis function method [9]. In this paper, Taylor series least square method is used with explicit time discretisation scheme.

Solving the flows with high computational efficiency is another main objective in the Computa- 
tional Fluid Dynamics (CFD) community. To achieve this aim, different research works have been carried out and numerous methods by different complexities were presented. It is obvious that the quality of the numerical solutions of Euler and Navier-Stokes equations is dependent upon the quality of the grid, especially in the critical zones of the flow domain, such as shock waves, stagnation points, and shear layer areas. Grid adaptation is one of the known answers to this problem. There are several methods presented in the literature for structured and unstructured grid adaptations $[10,11]$. But, only few works are reported on adaptive cloud meshless methods [12-15]. Compared with the mesh based algorithms, meshless methods are more advantageous, especially in the moving and large deformations. The reason is that replacing and moving points are much simpler than changing or replacing the edges and volumes. Another attractive property of meshless methods is the ability of adding and subtracting nodes from the pre-existing nodes [16]. There are different grid adaptation methods that normally fall in three categories of grid movement, grid refinement/coarsening, and re-gridding strategies. Lohner et al. [17] tried to use adaptation scheme to achieve optimal least square coefficients in a meshless method. The least square coefficients only depended upon the coordinates of points and their neighbors. Wang, G. et al. [18] used a point replacement method to solve the unsteady flows by meshless method. In another work, Wang, H. et al. used Delaunay triangulation principles to replace points in the domain for solution of unsteady flow [19].

In this paper, three different adaptive meshless methods are presented for compressible flow calculations. In this investigation, the derivatives are calculated by using least square method based on Taylor series $[7,9]$.

\section{Governing flow equations}

In the present work, the unsteady Navier-Stokes equations are used in two dimensions as follows:

$$
\left[\frac{\partial \mathbf{w}}{\partial t}\right]+\left[\frac{\partial \mathbf{f}^{I}}{\partial x}+\frac{\partial \mathbf{g}^{I}}{\partial y}\right]=\frac{\mathrm{M}_{\infty}}{\operatorname{Re}_{\infty}}\left[\frac{\partial \mathbf{f}^{V}}{\partial x}+\frac{\partial \mathbf{g}^{V}}{\partial y}\right],
$$

where the vector $\mathbf{w}$ and the flux vectors $\mathbf{f}$ and $\mathbf{g}$ are defined as:

$$
\begin{aligned}
\mathbf{w}=\left(\begin{array}{c}
\rho \\
\rho u \\
\rho v \\
\rho E
\end{array}\right), & \mathbf{f}^{I}=\left(\begin{array}{c}
\rho u \\
\rho u u+P \\
\rho v u \\
\rho E u+P u
\end{array}\right), \\
\mathbf{g}^{I}=\left(\begin{array}{c}
\rho V \\
\rho u V \\
\rho v V+P \\
\rho E V+P v
\end{array}\right), & \mathbf{f}^{V}=\left(\begin{array}{c}
0 \\
\tau_{x x} \\
\tau_{x y} \\
u \tau_{x x}+v \tau_{x y}-q_{x}
\end{array}\right),
\end{aligned}
$$

$$
\mathbf{g}^{V}=\left(\begin{array}{c}
0 \\
\tau_{x y} \\
\tau_{y y} \\
u \tau_{x y}+v \tau_{y y}-q_{y}
\end{array}\right)
$$

$u$ and $v$ are the flow velocity components in $x$ and $y$ directions, respectively. While $U$ and $V$ represent the relative velocity components and $p, \rho$, and $E$ are the pressure, density, and total energy, respectively, in the domain. For a perfect gas, we can have:

$$
p=(\gamma-1) \rho\left[E-\frac{u^{2}+v^{2}}{2}\right] .
$$

In this equation, $\gamma$ is the ratio of the specific heats [20].

\section{Meshless method}

To approximate the derivatives, the least-square scheme based on Taylor formula is used. By considering a group of points that are neighbors of point $i$ (Figure 1) and $\phi_{i j}$ as a value of any parameter at the mid-point of each pair point $i j$ ( $i$ and $j$ are neighbors), one can have $[7,21]$ :

$$
\begin{aligned}
& \left(\frac{\partial \phi}{\partial x}\right)_{i} \Delta x_{i j}+\left(\frac{\partial \phi}{\partial y}\right)_{i} \Delta y_{i j}=\Delta \phi_{i j}, \\
& \Delta x_{i j}=x_{j}-x_{i}, \quad \Delta y_{i j}=y_{j}-y_{i}, \\
& \Delta \phi_{i j}=\phi_{j}-\phi_{i} .
\end{aligned}
$$

It is notable that the neighbors of each point are the points which are in the influence domain of that point. This influenced region can be defined as a circle with the radius that can be different in the domain. By developing Eq. (4) for all neighbor points related to point $i$, the following matrix can be achieved [7]:

$$
\left[\begin{array}{cc}
\omega_{i 1} \Delta x_{i 1} & \omega_{i 1} \Delta y_{i 1} \\
\cdots & \cdots \\
\omega_{i m} \Delta x_{i m} & \omega_{i m} \Delta y_{i m}
\end{array}\right]\left[\begin{array}{c}
\left.\frac{\partial \phi}{\partial x}\right|_{i} \\
\left.\frac{\partial \phi}{\partial y}\right|_{i}
\end{array}\right]=\left[\begin{array}{c}
\omega_{i 1} \Delta \phi_{i 1} \\
\cdots \\
\omega_{i m} \Delta \phi_{i m}
\end{array}\right]
$$

where $\omega_{i j}$ is an arbitrary weighting factor. In this investigation, $\omega_{i j}$ is equal to the inverse of the distance

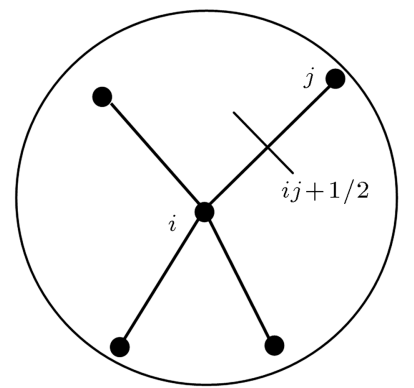

Figure 1. Schematic of point and its neighbors. 
between points $i$ and $j$. If the least-squares method is applied to solve Eq. (5), then the following equations for calculating the derivatives can be achieved $[9,21]$ :

$$
\left.\frac{\partial \phi}{\partial x}\right|_{i}=\sum_{j=1}^{m} a_{i j} \Delta \phi_{i j},\left.\quad \frac{\partial \phi}{\partial y}\right|_{i}=\sum_{j=1}^{m} b_{i j} \Delta \phi_{i j} .
$$

In this equation, $a_{i j}$ and $b_{i j}$ are least-square coefficients. These coefficients can be calculated as follows $[9,21]$ :

$a_{i j}=\frac{\omega_{i j} \Delta x_{i j} \sum_{k=1}^{m} \omega_{i k} \Delta y_{i k}{ }^{2}-\omega_{i j} \Delta y_{i j} \sum_{k=1}^{m} \omega_{i k} \Delta x_{i k} \Delta y_{i k}}{\sum_{k=1}^{m} \omega_{i k} \Delta x_{i j}{ }^{2} \sum_{k=1}^{m} \omega_{i k} \Delta y_{i k}{ }^{2}-\left(\sum_{k=1}^{m} \omega_{i k} \Delta x_{i k} \Delta y_{i k}\right)^{2}}$,
$b_{i j}=\frac{\omega_{i j} \Delta y_{i j} \sum_{k=1}^{m} \omega_{i k} \Delta x_{i k}{ }^{2}-\omega_{i j} \Delta x_{i j} \sum_{k=1}^{m} \omega_{i k} \Delta x_{i k} \Delta y_{i k}}{\sum_{k=1}^{m} \omega_{i k} \Delta x_{i j}{ }^{2} \sum_{k=1}^{m} \omega_{i k} \Delta y_{i k}{ }^{2}-\left(\sum_{k=1}^{m} \omega_{i k} \Delta x_{i k} \Delta y_{i k}\right)^{2}}$.

By using these equations in the governing Eq. (1), a semi-discrete form of the Navier-Stokes equations at point $i$ can be presented as follows:

$$
\begin{aligned}
{\left[\frac{\partial \mathbf{w}_{i}}{\partial t}\right] } & +\left[\sum_{j=1}^{m} a_{i j} \Delta \mathbf{f}_{i j}^{I}+\sum_{j=1}^{m} b_{i j} \Delta \mathbf{g}_{i j}^{I}\right] \\
& =\frac{\mathrm{M}_{\infty}}{\operatorname{Re}_{\infty}}\left[\sum_{j=1}^{m} a_{i j} \Delta \mathbf{f}_{i j}^{V}+\sum_{j=1}^{m} b_{i j} \Delta \mathbf{g}_{i j}^{V}\right]
\end{aligned}
$$

where $\Delta \mathbf{f}_{i j}=\mathbf{f}_{j}-\mathbf{f}_{i}$ and $\Delta g_{i j}=g_{j}-g_{i}$. By considering $H=a f+b g$ for each pair of points, flux can be calculated similar to flux calculation in mesh-based methods. So, Eq. (8), by substituting the direct flux, becomes $[9,21]$ :

$$
\left(\frac{\partial \mathbf{w}_{i}}{\partial t}\right)+\sum_{j=1}^{m} \Delta \mathbf{H}_{i j}=0, \quad \Delta \mathbf{H}_{i j}=\mathbf{H}_{j}-\mathbf{H}_{i} .
$$

The kind of discretization is central, in which the solution is unstable. To overcome this problem and diminish the oscillations, an artificial dissipation method is used [22].

$$
\begin{gathered}
\left(\frac{\partial \mathbf{w}_{i}}{\partial t}\right)+2 \sum_{j=1}^{m} \Delta \mathbf{H}_{i j+1 / 2}^{I}-\frac{2 \mathrm{M}_{\infty}}{\operatorname{Re}_{\infty}}\left[\sum_{j=1}^{m} \Delta \mathbf{H}_{i j+1 / 2}^{V}\right] \\
-D_{i}=0
\end{gathered}
$$

where $D_{i}$ is the dissipation term which is added to equations. In this context, the Jameson-SchmidtTurkel (JST) scheme for artificial dissipation term is used [20,22]:

$$
\begin{gathered}
\mathbf{D}_{i}=\left[\left(\nabla\left(\varepsilon^{(2)} \lambda\right) \nabla \mathbf{w}\right)-\left(\nabla^{2}\left(\varepsilon^{(4)} \lambda\right) \Delta^{2} \mathbf{w}\right)\right]_{i} \\
{\left[\left(\nabla\left(\varepsilon^{(2)} \lambda\right) \nabla \mathbf{w}\right)\right]_{i}} \\
=2 \sum_{j=1}^{m}\left(\zeta_{i j}\left(\varepsilon^{(2)} \lambda\right)_{j+1 / 2}\left(\mathbf{w}_{j}-\mathbf{w}_{i}\right)\right), \\
{\left[\left(\nabla^{2}\left(\varepsilon^{(4)} \lambda\right) \Delta^{2} \mathbf{w}\right)\right]_{i}} \\
=2 \sum_{j=1}^{m}\left(\zeta_{i j}\left(\varepsilon^{(4)} \lambda\right)_{j+1 / 2}\left(\Delta^{2} \mathbf{w}_{j}-\Delta^{2} \mathbf{w}_{i}\right)\right), \\
\zeta_{i j}=\frac{\sqrt{a_{i j}^{2}+b_{i j}^{2}}}{\left\|\vec{L}_{i j}\right\|}, \\
\Delta^{2} \mathbf{w}_{i}=\sum_{k=1}^{m}\left(\mathbf{w}_{k}-\mathbf{w}_{i}\right)=\sum_{k=1}^{m}\left(\mathbf{w}_{k}\right)-m \mathbf{w}_{i},
\end{gathered}
$$

here, $\varepsilon^{(2)}$ and $\varepsilon^{(4)}$ are local adaptive coefficients. In these coefficients, the pressure gradient as a sensor is used to find sharp gradients in the domain, such as shocks [20,21].

By developing Eq. (10) for all nodes in the domain, the following equation can be obtained [9]:

$$
\frac{\partial \mathbf{w}_{i}}{\partial t}+\mathbf{R}_{i}(\mathbf{w})=0
$$

where:

$$
\begin{aligned}
\mathbf{R}_{i}(\mathbf{w})= & 2 \sum_{j=1}^{m} \Delta \mathbf{H}_{i j+1 / 2}^{I}(\mathbf{w}) \\
& -2 \frac{\mathrm{M}_{\infty}}{\operatorname{Re}_{\infty}}\left[\sum_{j=1}^{m} \Delta \mathbf{H}_{i j+1 / 2}^{V}(\mathbf{w})\right]-\mathbf{D}_{i} .
\end{aligned}
$$

For the first term in Eq. (12), time marching methods are usually used. In this paper, the explicit four-stage Runge-Kutta (R-K) scheme is used as follows:

$$
\begin{aligned}
& \mathbf{w}^{(0)}=\mathbf{w}^{(n)} \\
& \mathbf{w}^{(1)}=\mathbf{w}^{(0)}-\alpha_{1} \Delta t \mathbf{R}\left(\mathbf{w}^{(0)}\right) \\
& \cdots \cdots \\
& \mathbf{w}^{(m)}=\mathbf{w}^{(0)}-\alpha_{m} \Delta t \mathbf{R}\left(\mathbf{w}^{(m-1)}\right) \\
& \mathbf{w}^{(n+1)}=\mathbf{w}^{(m)},
\end{aligned}
$$

where $m$ is the order of R-K that is taken 4 in this investigation and the coefficient $\alpha$ can be achieved from [20]. Local time stepping is used in this investigation [20,21]. At solid boundary, mass or other fluxes reflect the solid body $[7,20,21]$. To increase the accuracy in the solid boundary, ghost point method is applied in this investigation $[7,23]$. In the far field, Riemann invariants method is used [21]. 


\section{Point adaptation methods}

Capturing the flow phenomenon in the domain plays an important role in numerical fluid calculations. One way to increase the accuracy of the results, especially in the critical zones of the domain, is flow adaptation. In this paper, three different point adaptation methods of point movement, point refinement/coarsening, and neighbor adaptation are developed. follow:

The general steps of adaptation method are as

a) Generating the initial point distribution within the domain;

b) Flow solution using the initial point distribution;

c) Calculation of the local errors;

d) Adapting the point (or neighbor) distribution based on the local error;

e) Interpolating the flow variables on the new points;

f) Solving the flow over the new point distribution.

The point adaptation methods are implemented at step (d) of the above steps. It should be noted that the adaptation procedure is performed only once during the above steps, since it was found to be sufficient for acceptable results. The flowchart of adaptation steps is shown in Figure 2. The implementation details of these methods are explained in the following sections.

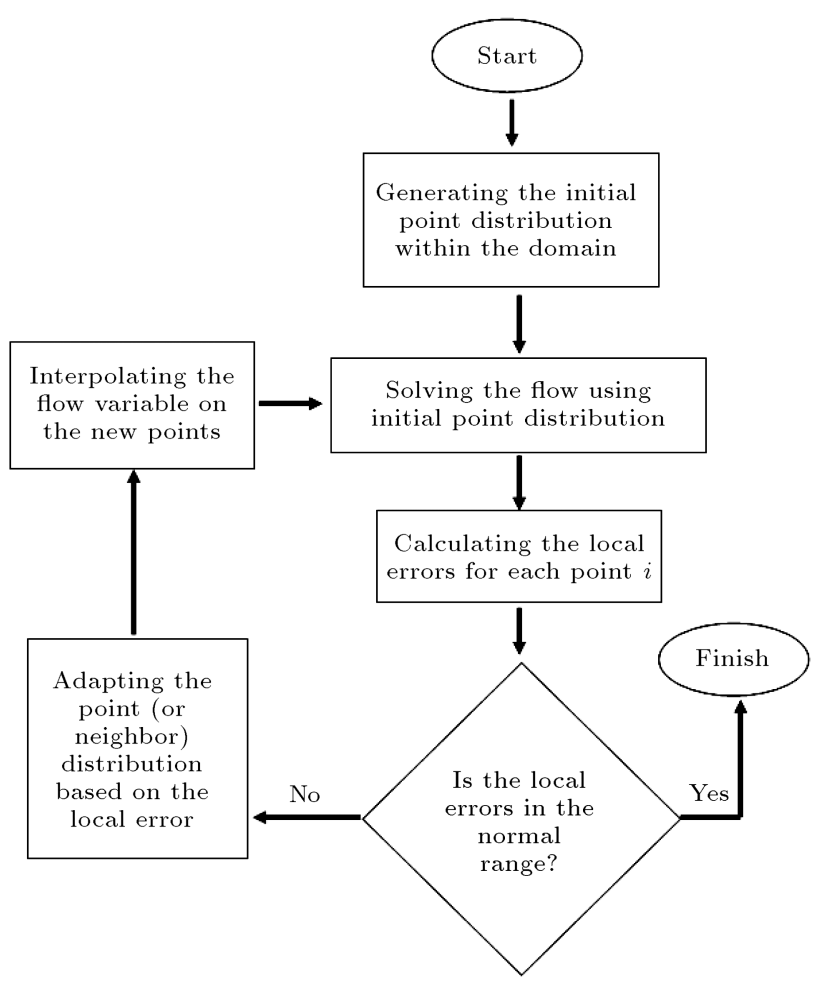

Figure 2. The flowchart of adaptation method.

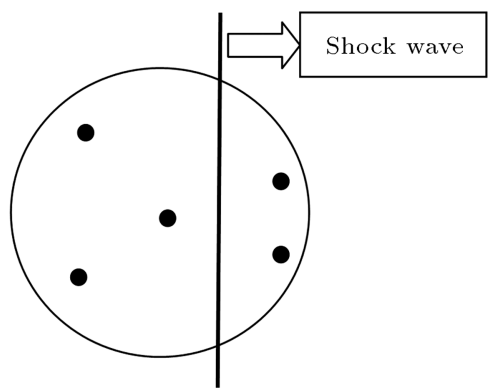

(a) Before adaptation

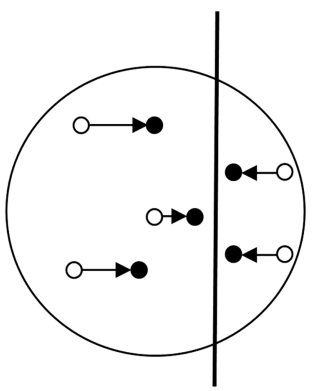

(b) After adaptation
Figure 3. Point movement strategy.

\subsection{Point movement}

In the point movement strategy used in this work, the points are allowed to move towards the higher gradient regions (i.e., shock wave) of the flow domain according to a classical spring analogy scheme $[24,25]$. In this method, the total number of points is fixed in the domain and points are replaced according to the local error (Figure 3). Therefore, by using spring method, the coordinates of the point locations can be obtained by iteratively calculating the following equations:

$$
\begin{aligned}
& x_{A}^{m+1}=x_{A}^{m}-\frac{c \sum_{n=1}^{N} w_{n}\left(x_{A}^{m}-x_{n}^{m}\right)}{\sum_{n=1}^{N} w_{n}}, \\
& y_{A}^{m+1}=y_{A}^{m}-\frac{c \sum_{n=1}^{N} w_{n}\left(y_{A}^{m}-y_{n}^{m}\right)}{\sum_{n=1}^{N} w_{n}},
\end{aligned}
$$

where $c$ is the relaxation factor chosen between 0.5 and 1.0, and $w_{n}$ is the weight function defined to capture the features of the flow. For transonic flows considered in this work, the main flow feature presented is shock wave; thus, the suitable detection parameter is calculated as:

$$
w_{n}=\frac{\left|p_{A}-p_{n}\right|}{\left|p_{A}+p_{n}\right|} \sqrt[4]{\left(x_{A}-x_{n}\right)^{2}+\left(y_{A}-y_{n}\right)^{2}} .
$$

In order to have the best quality of point distribution, the above equations are performed several times in a loop. Normally, 10 to 20 iterations are sufficient and give acceptable results. It is notable that only one adaptation cycle is performed in this work as it was found to be sufficient for optimum results.

\subsection{Point refinement/coarsening}

In this method, the new points are added in the areas where flow features have occurred. Thus, the number of points is not fixed. One of the advantages of this method is high quality of point distribution in different zones. At first, the error $\left(e_{k}\right)$ for each pair node is 


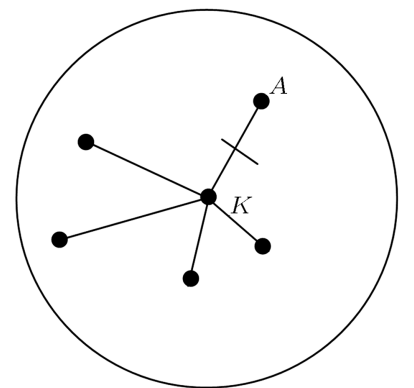

Figure 4. A sample point $K$ and its neighbors.

calculated, as shown in Figure 4, to find if a new point is needed for addition or not. To calculate the error indicator $e_{k}$, the non-dimensional form of the pressure $(p)$ gradient is chosen in the following form for each pair of nodes $(K, A)$ :

$$
e_{K}=\frac{\left|p_{A}-p_{K}\right|}{\left|p_{A}+p_{K}\right|} \sqrt[4]{\left(x_{A}-x_{K}\right)^{2}+\left(y_{A}-y_{K}\right)^{2}}
$$

A function of the distance between two points $(A, K)$ is considered in the above formula in order to prevent over-refinement of the points near the discontinuities (i.e., shock waves). Then, the average error (M) is calculated for the domain as:

$$
\mathrm{M}=\frac{1}{n} \sum_{k=1}^{n}\left|e_{k}\right|
$$

where $n$ is the number of nodes in the domain. By defining $\mathrm{M}$ and $e_{k}$, the standard deviation $\sigma$ can be calculated as follows:

$$
\sigma=\sqrt{\frac{\sum_{k=1}^{n}\left(\left|e_{k}-\mathrm{M}\right|\right)^{2}}{n}} .
$$

To find if a pair point is to be refined or not, the statistical Relation (20) should be checked. If this equation is satisfied, then a point should be added in between the pair point $(K, A)$.

$$
e_{k} \geq \mathrm{M}+\alpha \sigma
$$

Again, for coarsening, the following equation should be checked:

$$
e_{k} \leq \mathrm{M}-\alpha \sigma
$$

The amount of $\alpha$ is based on the problem and is different in coarsening and refinement [26,27]. In the present work, the values of 0.1 and 0.25 are used for point refinement and point coarsening, respectively.

The adaptive process, at first, starts with coarsening, and the points that satisfy Eq. (21) are omitted. It is important that when one point is deleted, its neighbors are not allowed to be deleted at this step,

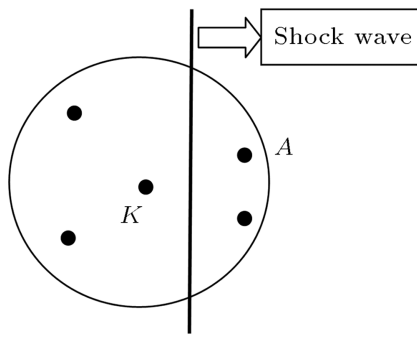

(a) Before adaptation

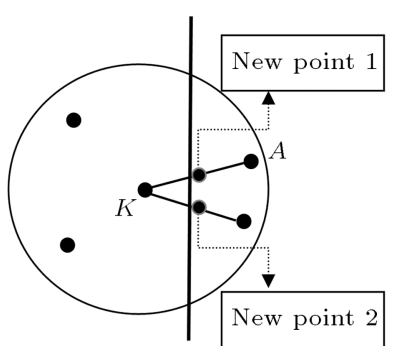

(b) After adaptation
Figure 5. Point refinement method.

because it may decrease the accuracy of solution in this area. After performing the coarsening step, the neighboring data structure should be updated. In the next step, the refinement process is performed. The added point is situated in the middle of each pair point selected for refinement, as sketched in Figure 5, and its neighbors are defined from the neighbors of two original points.

\subsubsection{Neighbor region adaptation}

The next method is based on the main privilege of meshless methods that there is no edge between points. This privilege can be resulted in that as different points in the domain based on the flow complexity are applied as neighbors. For example, across critical zones of the flow field such as shock waves, the number of neighbors for each point is increased. Thus, in this adaptation method, the number of points is fixed in the domain while the neighbors of points could change according to the flow features identified by calculating the local errors.

In this method, the statistical parameters are applied, too. The error indicator $\left(e_{k}\right)$, average error M, and the standard deviation $(\sigma)$ are calculated through Relation (20). If this relation is satisfied for each pair point $(K, A)$, which means the error is higher than the predefined value, then a point in the domain should be added to the neighbor list of the original point $K$, so that the accuracy is preserved in this region. The added neighbor is the nearest point to the neighbor point $A$ (Figure 6 ). It is clear that this method has the advantage that without changing the number and position of the points, it increases the accuracy and efficiency of the calculations.

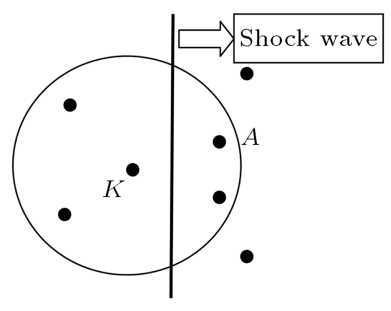

(a) Before adaptation

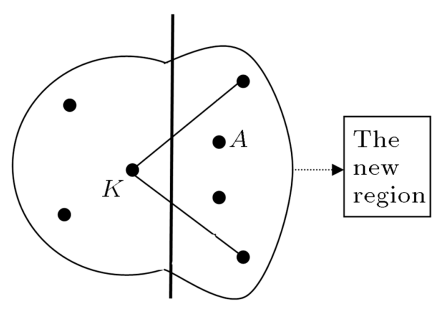

(b) After adaptation
Figure 6. Neighbor region adaptation. 


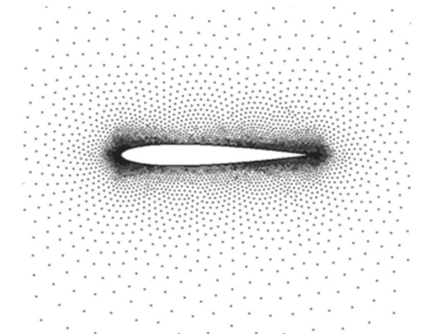

(a) Without adaptation (number of points is 5375 )

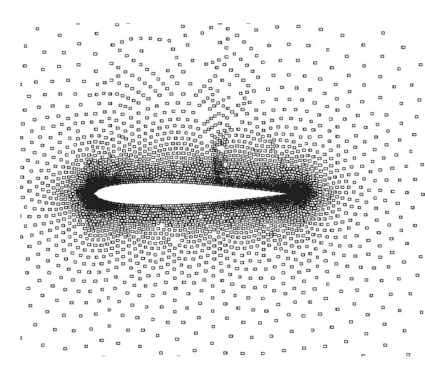

(c) With point refinement (number of points is 5423)

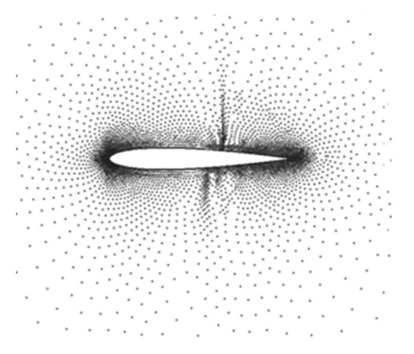

(b) With point movement

(d) With point refinement+ movement (number of points
is 5382 )

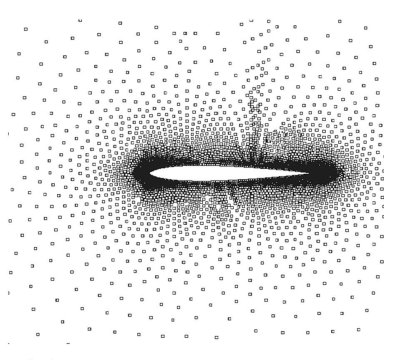

Figure 7. Point distribution around NACA 0012 at $\mathrm{M}=0.8$ and $\mathrm{AOA}=1.25$.

\section{Results}

In order to show the capabilities of the point adaptation methods presented in this paper, three different test cases are applied.

The first case is an inviscid flow at transonic flow conditions of $\mathrm{M}=0.8$ and $\mathrm{AOA}=1.25$. The initial point distribution is shown in Figure 7(a). The point cloud after flow adaptation using point movement method is shown in Figure $7(\mathrm{~b})$. There are 5375 points in the domain. The number of points on the solid boundary is 282 . The point cloud after point enrichment/coarsening is shown in Figure $7(\mathrm{c})$. It is notable that the number of points after this type of adaptation is 5403. In Figure $7(\mathrm{~d})$, the point distribution after implementation of both methods of point movement and point refinement is shown. It is notable that the number of points here is less than that in the previous case, where only point refinement was used. The pressure contours are compared in Figure 8(a) and (b). As illustrated in this figure, the shock wave is well resolved with adapted distribution of points in comparison with the initial distribution (without adaptation).

The surface pressure coefficient distributions are shown for the adapted and un-adapted point distributions in Figure 8(c), comparing them with the finite volume results of [20] that used the similar discretization method as the current meshless method. As illustrated, the adapted results are in better agreement with the referenced finite volume data, especially in capturing the shock wave. The convergence histories of adapted and un-adapted results are compared in Figure 8(d) to show the computational efficiency of each adaptation method. It is clear from this figure that the point movement, point enrichment/coarsening, and neighbor adaptation methods can individually decrease the computational time by $35 \%, 30 \%$, and $10 \%$, respectively. Additionally, the combination of three methods reduces the computational time by about $60 \%$. As it is obvious, the combination of methods has better convergence rate than each adaptation method, separately. The reason is that in the combination method, not only the number of points is optimized but also they are situated in better places with optimized neighbors. The computations are carried out on a Pentium PC Dual core with $2.00 \mathrm{GHz}$ speed. The results indicate that in the third method, the added neighbors increase the accuracy of the derivatives by balancing the neighbors of each point (Figure 9).

The second test case is a transonic laminar flow around NACA0012 airfoil at $\mathrm{M}=0.8, \mathrm{Re}=500$, and $\mathrm{AOA}=10$ degrees. This is a well-known separated flow that has been used for validation of numerical methods. The initial point distribution has 13233 points of which 364 points are in the solid boundary. In Figure 10(a) and (b), the point distribution before and after adaptation is shown. The number of points after adaptation is 13421 which is only less than 2 percent more than the initial distribution. In Figure 10(c) and (d), Mach number contours before and after adaptation are shown. As it is obvious, smooth results are achieved after adaptation in comparison with unadapted results. In this case, Mach number variation is chosen as the error indicator $e_{k}$ as follows:

$$
e_{k}=\mathrm{M}_{A}-\mathrm{M}_{k} \text {. }
$$

In this equation, $A$ and $K$ are pair points. The surface pressure coefficient distribution and the convergence histories of the results are shown in Figure 11. As illustrated, good agreements are achieved for surface pressure coefficient distribution in comparison with the Control Volume method results [20]. As illustrated, about $60 \%$ reduction in computational time is achieved in this case as compared with the un-adapted results. This exhibits similar computational efficiency to that of the previous inviscid case.

The next case is an unsteady flow around oscillating NACA0012 airfoil that is considered to investigate the capabilities of the adapted methods for such complicated problems in which the flow features are moving. Point distribution is the same as that in the first case. The movement of solid boundary changes the situation of the points. The flow condition is Mach number 0.755 and the periodic angle around the quarter chord by considering $\alpha_{m}$ as a mean angle, $\alpha_{0}$ as the oscillation amplitude, and $\omega$ as the frequency of the system can be achieved as follows: 


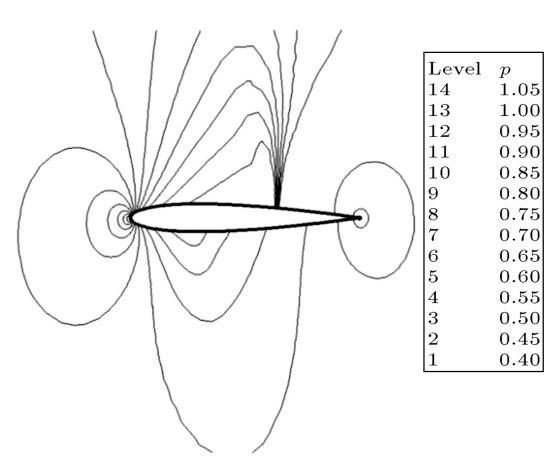

(a) Pressure contours without adaptation

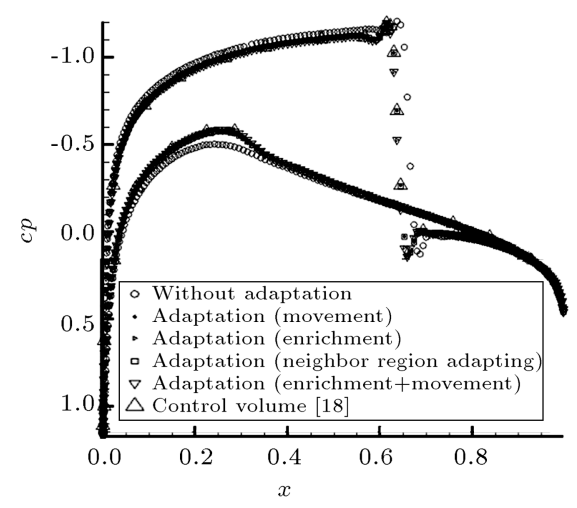

(c) The surface pressure coefficient distribution

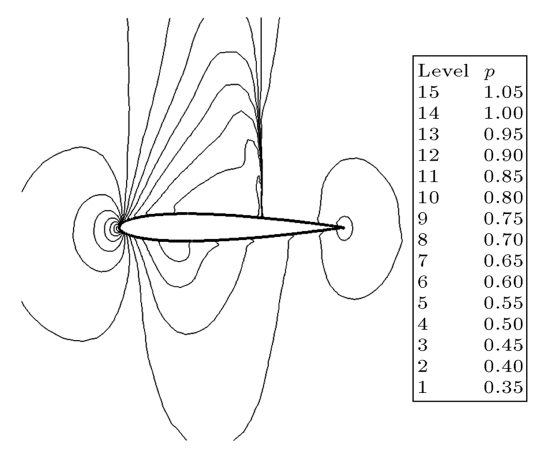

(b) Pressure contours after adaption (refinement+movement)

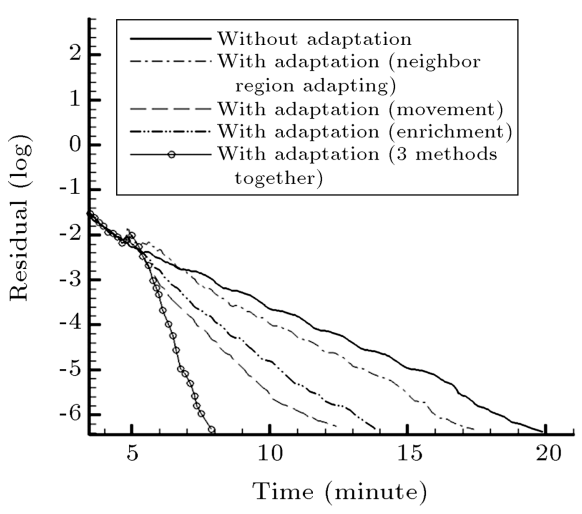

(d) The convergence history

Figure 8. Results for the flow around NACA 0012 at $\mathrm{M}=0.8$ and $\mathrm{AOA}=1.25$.

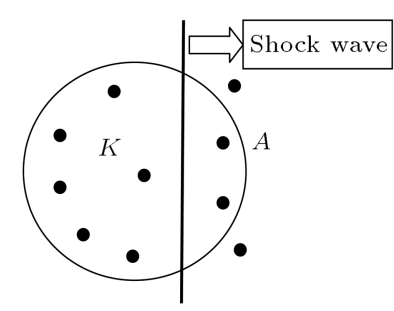

(a) Before adaptation

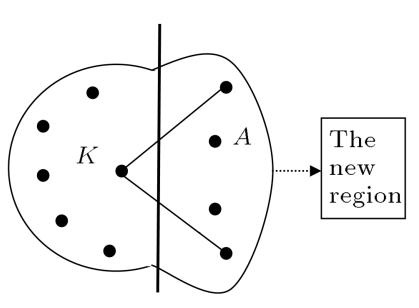

(b) After adaptation

Figure 9. Balancing the neighbors in the third method (neighbor region adaptation method).

$$
\alpha(t)=\left(\alpha_{m}+\alpha_{0} \sin (\omega t)\right),
$$

where $\alpha_{m}$ is chosen $0.016, \alpha_{0}$ is taken as 2.51 , and $\omega$ is calculated as follows:

$$
\omega=\frac{2 k U_{\infty}}{c}
$$

where $U_{\infty}$ is velocity in the far field, $c$ is the chord of the airfoil, and $k$ is a reduced frequency which is equal to 0.814 in this work. The point distribution and pressure contours of adapted results for three different periodic angles are shown in Figure 12. As mentioned before, the number of points within the domain remains constant when using point movement method while the number of points is changed in the

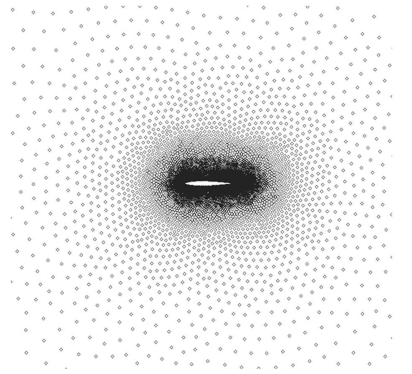

(a) Point distribution before adaptation

(b) Point distribution after adaptation (refinement+movement; number of points is 13421)

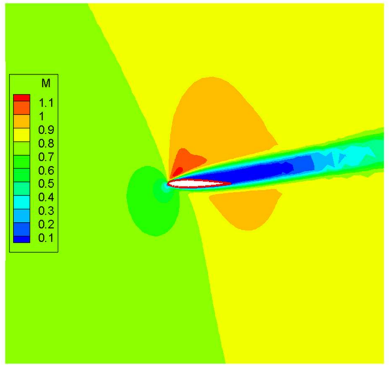

(c) Mach number contours before adaptation

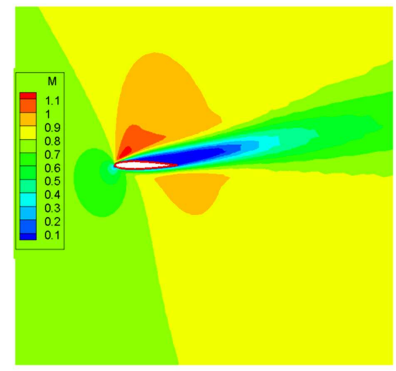

(d) Mach number contours after adaptation
Figure 10. The point distribution and Mach number contours before and after adaption for flow around NACA $0012, \mathrm{M}=0.8, \mathrm{AOA}=10$, and $\operatorname{Re}=500$. 


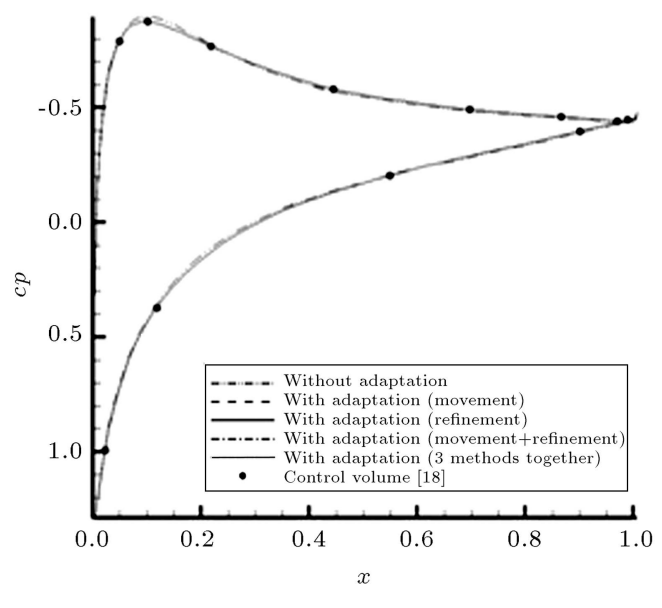

(a) The surface pressure coefficient distribution

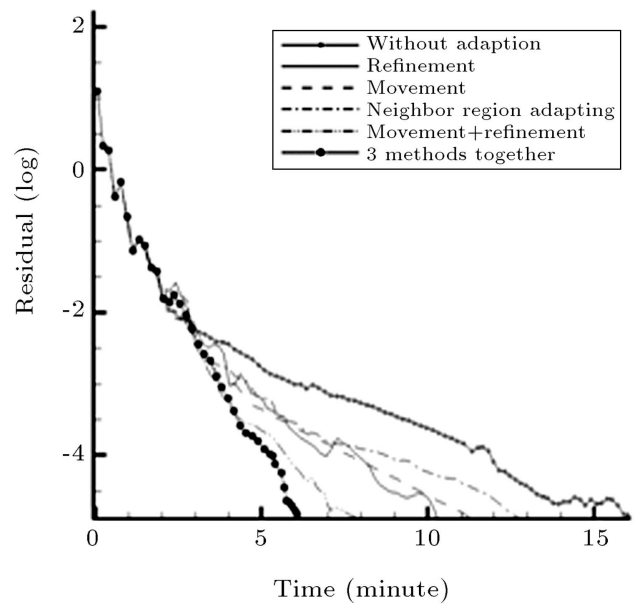

(b) Convergence history

Figure 11. The surface pressure coefficient distribution and convergence history for flow around NACA $0012, \mathrm{M}=0.8$, $\mathrm{AOA}=10$, and $\mathrm{Re}=500$.

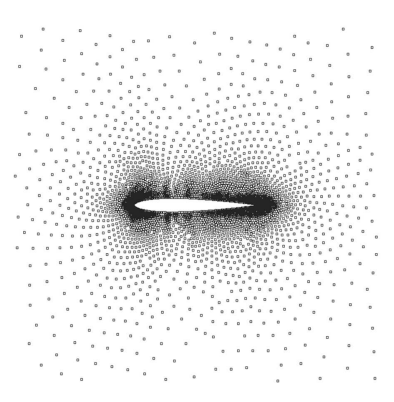

(a) Point distribution at $\mathrm{AOA}=0$ (5325 points)

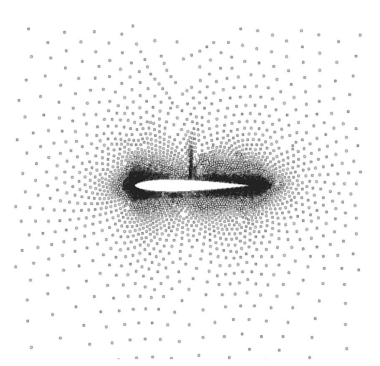

(c) Point distribution at $\mathrm{AOA}=2(5334$ points $)$

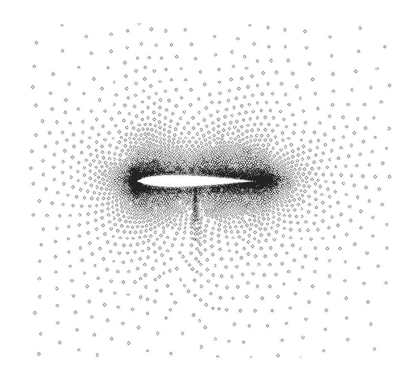

(e) Point distribution at $\mathrm{AOA}=-2(5343$ points $)$

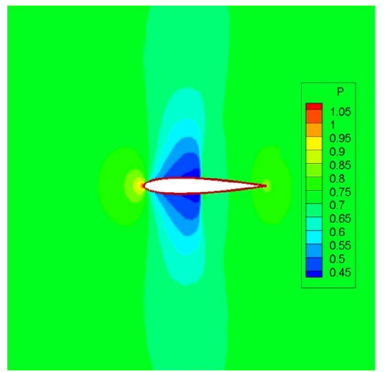

(b) Pressure contour at $\mathrm{AOA}=0$

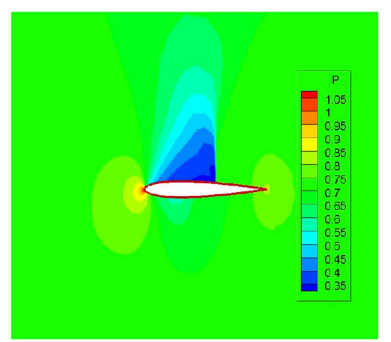

(d) Pressure contour at $\mathrm{AOA}=2$

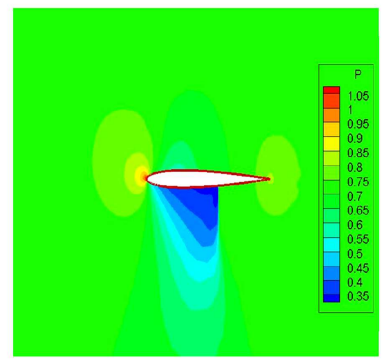

(f) Pressure contour at $\mathrm{AOA}=-2$

Figure 12. Point distribution and pressure contours after adaptation (refinement+movement) at Mach number of 0.755 . point refinement method based on the flow features. In this case, the numbers of points for the combination method are 5325, 5334, and 5343, for $\mathrm{AOA}=0$, $\mathrm{AOA}=2, \mathrm{AOA}=-2$, respectively. While the numbers of points for refinement method are higher than those for the combination method (5401, 5432, 5412 for $\mathrm{AOA}=0, \mathrm{AOA}=2, \mathrm{AOA}=-2$, respectively), which can increase the computational cost and decrease the convergence rate. It is clear from this figure that the moving shock wave has suitably been captured and refined by the presented adaptation methods. The normal force coefficients and pitching moment coefficients obtained from inviscid flow solution with different adaptation methods are shown in Figure 13 in comparison with the experimental data of AGARD [28].

\section{Conclusions}

In this paper, three different adaptive meshless methods were presented. An explicit meshless method based on the least square scheme was used. Taylor series based on the least square method was used for calculating the derivatives at each point. Three different adaptive methods were developed, including point movement, point refinement/coarsening, and neighbor adaptation, for meshless calculation of steady and unsteady fluid flows. The results were compared with those of other reliable numerical methods and it was shown that the accuracy of the adapted results for each method and the combination of methods was improved. In addition, the computational cost and convergence time were discussed in different flow situations. The results showed that these adaptation methods can decrease the computational cost by about $60 \%$ as compared with un-adapted method. 


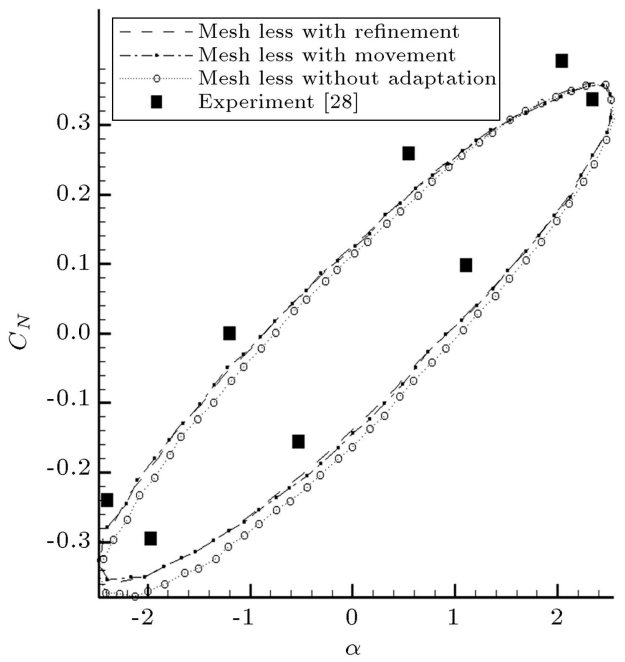

(a) Normal force coefficient

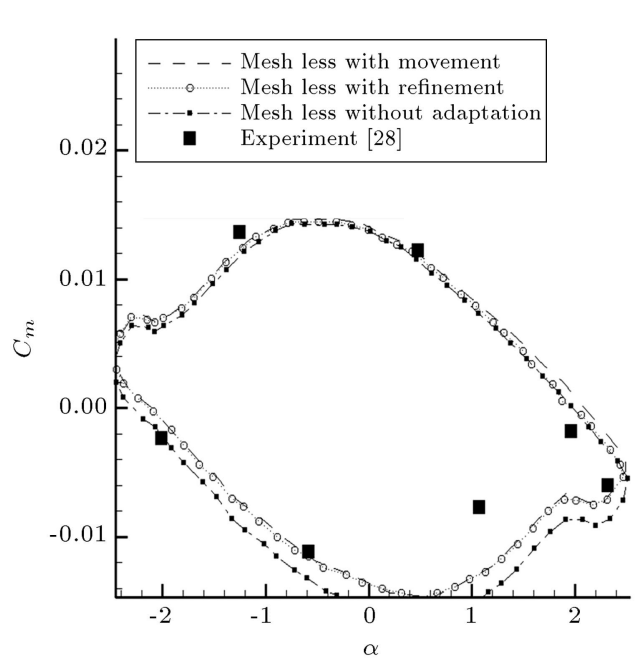

(b) Pitching moment coefficient

Figure 13. Aerodynamic coefficients for the unsteady flow around NACA 0012, and $M=0.755$.

\section{References}

1. Jameson, A., Baker, T.J. and Weatherill, N.P. "Calculation of inviscid transonic flow over a complete aircraft", AIA A Paper, AIAA-86-0103 (1986).

2. Liu, G.R. and Gu, Y.T., An Introduction to Mesh Free Methods and Their Programming, Springer, The Netherlands (2005).

3. Nguyen, V.P., Rabczuk, T., Bordas, S. and Marc Duflot M. "Mesh less methods: A review and computer implementation aspects", Mathematics and Computers in Simulation, 79, pp. 763-813 (2008).

4. Ding, H., Shu, C., Yeo, K.S. and Xu, D. "Simulation of incompressible viscous flows past a circular cylinder by hybrid FD scheme and mesh-less least squarebased finite difference method", Computer Methods in Applied Mechanics and Engineering, 193, pp. 727-744 (2004).

5. Deshpande, S.M., Praveen, C. and Ramesh, V. "Theory and application of 3-D LSKUM based on entropy variables", International Journal for Numerical Methods in Engineering, 40(1), pp. 47-62 (2002).

6. Batina, J.T. "A gridless Euler/Navier-Stokes solution algorithm for complex aircraft applications", AIAA Paper, No. 93 (1993).

7. Jahangirian, A. and Hashemi, Y. "An efficient implicit mesh-less method for compressible flow calculations", International Journal for Numerical Methods in Flu$i d s, 67(6)$, pp. 754-770 (2011).

8. Katz, A. and Jameson, A. "Multicloud: multigrid convergence with a meshless operator", Journal of Computational Physics, 228, pp. 5237-5250 (2009).

9. Katz, A. and Jameson, A. "A comparison of various meshless schemes within a unified algorithm", 47th AIAA Aerospace Sciences Meeting and Exhibit, Orlando, Florida, AIAA paper 2009-0596 (2009).

10. Holmes, D.G. and Lamson, S.H. "Adaptive triangular meshes for compressible flow solutions", Numerical
Grid Generation in Computational Fluid Dynamics, Pineridge Press (1986).

11. Jahangirian, A. and Johnston, L.J. "Automatic generation of adaptive unstructured grids for viscous flow applications", 5th International Conference on Numerical Grid Generation in CFD, Mississippi State University (1996).

12. Kim, H.G. and Atluri, S.N. "Arbitrary placement of secondary nodes, and error control, in the meshless local Petrov-Galerkin (MLPG) method", Computer Modeling in Engineering \& Sciences, 1(3), pp. 11-32 (2000).

13. Amani, J., Saboor Bagherzadeh, A. and Rabczuk, T. "Error estimate and adaptive refinement in mixed discrete least squares meshless method", Mathematical Problems in Engineering, Article ID 721240, 16 pages, 2014. doi:10.1155/2014/721240 (2014).

14. Angulo, A., Pérez Pozo, L. and Perazzo, F. "A posteriori error estimator and an adaptive technique in meshless finite points method", Engineering Analysis with Boundary Elements, 33(11), pp. 1322-1338 (2009).

15. Armando Duarte, C. and Tinsley Oden, J. "An h-p adaptive method using clouds", Computer Methods in Applied Mechanics and Engineering, 139(4), pp. 237262 (1996).

16. Ortega, E., Onate, E., Idelsohn, S. and Flores, R. "A meshless finite point method for three-dimensional analysis of compressible flow problems involving moving boundaries and adaptivity", International Journal for Numerical Methods in Fluids, 73(4), pp. 323-343 (2013).

17. Perazzo, F., Lohner, R. and Perez-Pozo, L. "Adaptive methodology for meshless finite point method", Advances in Engineering Software, 39, pp. 156-166 (2008). 
18. Wang, G., Sun, Y. and Ye, Z. "Gridless solution method for two-dimensional unsteady flow", Chinese Journal of Aeronautics, 18(1), pp. 8-14 (2005).

19. Wang, H., Chen, H.Q. and Periaux, J. "A study of gridless method with dynamic clouds of points for solving unsteady CFD problems in aerodynamics", Int. J. Numer. Meth. Fluids, 64(1), pp. 98-118 (2010).

20. Jahangirian, A. and Hadidoolabi, M. "Unstructured moving grids for implicit calculation of unsteady compressible viscous flows", International Journal for Numerical Methods in Fluids, 47(11), pp. 1107-1113 (2005).

21. Sattarzadeh, S. and Jahangirian, A. "3D implicit mesh-less method for compressible flow calculations", Journal of Scientia Iranica, 19(3), pp. 503-512 (2012).

22. Swanson, R.C., Radespiel, R. and Turkel, E. "On some numerical dissipation schemes", Journal of computational Physics, 147(2), pp. 518-544 (1998).

23. Hashemi, Y. and Jahangirian, A. "Adaptive Cartesian grid with mesh-less zone for compressible flow calculations", Computers \& Fluids, 54, pp. 10-17 (2012).

24. Batina, J.T. "Unsteady Euler algorithm with unstructured dynamic mesh for complex-aircraft aeroelastic analysis", AIA A Paper 89-1189 (1989).

25. Blom, F.J. "Considerations on the spring analogy", International Journal for Numerical Methods in Fluids, 32, pp. 647-668 (2000).

26. Ortega, E., Onate, E., Idelsohn, S. and Buachart,
C. "An adaptive finite point method for the shallow water equations", International Journal for Numerical Methods in Engineering, 88(2), pp. 180-204 (2011).

27. Holmes, D.G. and Connell, S.D. "Solution of the 2D Navier-Stokes equations on unstructured adaptive grids", AIA A paper 1989-1932-cp (1989).

28. Compendium of Unsteady Aerodynamic Measurements, AGARD 1982, R-702 (1982).

\section{Biographies}

Samad Sattarzadeh received an MS degree in Aerospace Engineering from Amirkabir University of Technology (AUT), Tehran, Iran, where he is currently a $\mathrm{PhD}$ degree student. His research interests include meshless methods.

Alireza Jahangirian received a BS degree from Amirkabir University of Technology (AUT), Tehran, Iran, in 1988 and an MS degree from Sharif University of Technology, Tehran, Iran, in 1992, both in Mechanical Engineering. He received his $\mathrm{PhD}$ degree from Manchester University, England, in 1997. In 1998, he joined the Department of Aerospace Engineering, AUT, where he is currently Associate Professor. His research interests include computational fluid dynamics, grid generation, and evolutionary aerodynamic optimization. 